\title{
A novel standardized distraction test to evaluate lower eyelid tension using three-dimensional stereophotogrammetry
}

\author{
Xiaoyi Hou ${ }^{1 \#} \wedge$, Alexander C. Rokohl ${ }^{1 \#} \wedge$, Marius M. Meinke ${ }^{1}$, Senmao Li ${ }^{1} \wedge$, Jinhua Liu ${ }^{1}$, Wanlin Fan ${ }^{1} \wedge$, \\ Ming $\operatorname{Lin}^{2} \wedge$, Renbing $\mathrm{Jia}^{2} \wedge$, Yongwei Guo ${ }^{3 *}$, Ludwig M. Heindl ${ }^{1,4 *} \wedge$ \\ ${ }^{1}$ Department of Ophthalmology, University of Cologne, Faculty of Medicine and University Hospital of Cologne, Cologne, Germany; ${ }^{2}$ Department \\ of Ophthalmology, Ninth People's Hospital, Shanghai Jiao Tong University School of Medicine, Shanghai, China; ${ }^{3}$ Eye Center, Second Affiliated \\ Hospital, School of Medicine, Zhejiang University, Hangzhou, China; ${ }^{4}$ Center for Integrated Oncology (CIO) Aachen-Bonn-Cologne-Duesseldorf, \\ Cologne, Germany \\ "These authors contributed equally to this work and both should be considered as first authors.
}

*These authors contributed equally to this work and both should be considered as senior authors.

Correspondence to: Ludwig M. Heindl, MD. Department of Ophthalmology, University of Cologne, Faculty of Medicine and University Hospital of Cologne, Kerpener Strasse 62, 50937 Cologne, Germany. Email: ludwig.heindl@uk-koeln.de; Yongwei Guo, MD. Eye Center, Second Affiliated Hospital, School of Medicine, Zhejiang University, 88 Jiefang Road, Hangzhou 310009, China. Email: yongwei-guo@zju.edu.cn.

\begin{abstract}
Background: Standardized pre-operative assessment of the lower eyelid tension is essential to determine the optimal surgical technique. However, quantitative analysis using the conventional distraction test is inaccurate and user-dependent. Our purpose was to introduce a novel, standardized three-dimensional distraction test for measuring lower eyelid tension and to determine its standard values in a Caucasian population.
\end{abstract}

Methods: In 94 participants (50 men and 44 women; age 21-85 years), a 15.9-g weighted eyelid hook was used to pull down the lower eyelid. Two three-dimensional images were acquired with a VECTRA M3 stereophotogrammetry device-one in the neutral position without a hook and the other in the distracted position with the eyelid hook. The images of all participants in both positions were measured twice by a single observer.

Results: There was no clinical $(>1 \mathrm{~mm})$ or statistically significant difference between the two repeated measurements of all the inter-landmark linear distances in both positions ( $\mathrm{P} \geq 0.05$, respectively). The mean distracted displacement between the neutral and distracted position for margin reflex distance was $5.50 \pm 1.53 \mathrm{~mm}$, without any age-specific difference $(\mathrm{P}=0.08)$; however, a significant gender-specific difference was observed as men had significantly greater displacement than women $(\mathrm{P}<0.001)$.

Conclusions: Our proposed standardized three-dimensional distraction test for assessing lower eyelid tension using an eyelid hook and a simple landmark-based system seems to provide high reliability. This novel and simple method might be helpful for the preoperative planning of eyelid surgeries.

Keywords: Distraction test; lower eyelid tension (LET); standard-weighed eyelid hook; three-dimensional stereophotogrammetry; landmark system

Submitted Aug 28, 2020. Accepted for publication Apr 01, 2021.

doi: 10.21037 /qims-20-1016

View this article at: http://dx.doi.org/10.21037/qims-20-1016

^ ORCID: Xiaoyi Hou, 0000-0001-5657-4984; Alexander C. Rokohl, 0000-0002-0224-3597; Senmao Li, 0000-0002-4844-4503; Wanlin Fan, 0000-0001-7143-6707; Ming Lin, 0000-0001-6467-4643; Renbing Jia, 0000-0001-6642-7451; Yongwei Guo, 0000-0001-9195-0770; Ludwig M. Heindl, 0000-0002-4413-6132. 


\section{Introduction}

The eyelids protect ocular structures, maintain the exterior cosmetic appearance, and balance the ocular surface with the suspended components, including a thin layer of skin, orbicularis oculi muscle, lower lid retractors, tarsus, and fibrous tissue (1). Aging is a major cause for decreased lower eyelid tension (LET) due to the vertical increase of the inferior lateral orbit distance and weak horizontal traction between the lateral canthus and the lower eyelid attachment (2). The lower eyelid margin is generally located 1-2 mm above the inferior corneoscleral limbus, and the lateral canthus is generally located $2-4 \mathrm{~mm}$ higher than the medial canthus $(1,3)$. With progressive laxity of horizontal tension, lower eyelid malposition may occur, which may cause not only unpleasant cosmetic diseases, such as ectropion and entropion, but also inappropriate eye exposure (3) and need to be corrected by surgery.

Pre-operative assessment of LET is particularly important to determine the optimal surgical technique (4). Previously, several studies have investigated the use of various devices to estimate LET (4-9). However, most of these instruments, such as the clamp placed on the eyelid margin and latex sensor inserted into the inferior fornix, were not feasible for use (4). Only the studies by Vihlen et al. (5) and Wilson et al. (6) attempted the in vivo evaluation of LET. Despite successful quantification of LET, the study results were considered to be inaccurate because of high variations in the measurements of displacement distances and the augmented assessment of palpebral tension (5).

Several studies have verified the accuracy and reliability of three-dimensional (3D) stereophotogrammetry for craniofacial anthropometric measurements (10). With the development of this new technique and the meticulous efforts required during eyelid reconstructive surgeries, high-resolution 3D stereophotogrammetry has been utilized in the periocular anthropometric evaluation of normal periocular parameters and the selection of optimal surgical technique (11). Recently, Stuchi et al. were the first to investigate the potential use of a $3 \mathrm{D}$ imaging system based on the distraction test (DT) to assess LET (12). Their results confirmed the accuracy and reliability of a functional test based on a 3D digital imaging system (12). However, their study only included participants with a narrow age range, and the exact LET was not quantitatively investigated using the conventional DT.

Hence, our study aimed to introduce a modified DT using a metal eyelid hook to assess the LET in different age groups and genders, and to investigate the feasibility by using a 3D digital imaging system to acquire standardized results of this novel, simple methods.

\section{Methods}

\section{Subjects}

Ninety-four Caucasian volunteers (50 men and 44 women, 94 eyes), aged $21-85$ years $(59.53 \pm 15.50$ years), were randomly recruited from the Department of Ophthalmology, University Hospital of Cologne. Only volunteers without any previous history of eyelid diseases, trauma, or surgeries were included in this study. Individuals with strabismus, morphological disorders, or long-term instillation of eye drops were excluded. Written informed consent was obtained from all volunteers. The study was approved by the institutional ethics board of the University of Cologne and informed consent was taken from all individual participants.

\section{Standardized tool: eyelid book}

A 15.9-g stainless-steel eyelid hook (Zhen Bang Medical Devices Co., Ltd., Anhui, China) with a head width, $1.0 \mathrm{~cm}$; body length, $15.0 \mathrm{~cm}$; and thickness, $1.0 \mathrm{~mm}$ (Figure 1) was used to pull down the lower eyelid in a standardized matter. Each participant was asked the comfort level when applying the eyelid hook. Participants who were not co-operative or extremely sensitive were excluded.

\section{D imaging system}

The VECTRA M3 3D Imaging System (Canfield Scientific, Inc., Parsippany, NJ, USA) was utilized for all image acquisitions. The Face Sculptor software of VECTRA was used for processing the $3 \mathrm{D}$ models, which were saved for further measurements and accurate analyses by the software of the VECTRA Analysis Module (VAM). A single experienced operator performed all image acquisition under the same conditions, following the manufacturer's guidelines.

\section{D image acquisition}

Two 3D images were acquired with the VECTRA M3 stereophotogrammetry device, each in the neutral position 

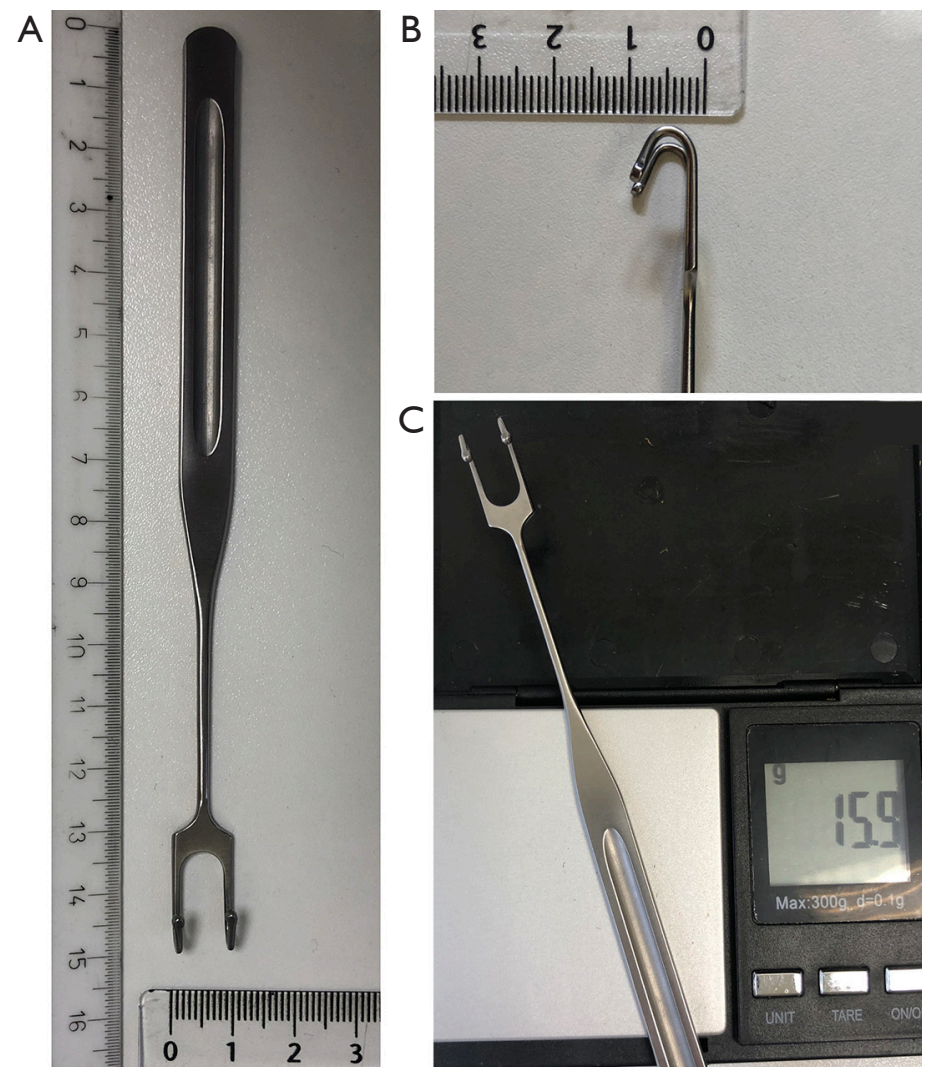

Figure 1 Parameters for the study tool, a stainless-steel eyelid hook (A,B,C). (A) The body length and head width of the eyelid hook is 15.0 and $1.0 \mathrm{~cm}$, respectively; (B) the thickness of the eyelid hook is $1.0 \mathrm{~mm}$; (C) the total weight of the eyelid hook is 15.9 grams.

(NP) and distracted position (DP) using the weighted eyelid hook to pull down the lower eyelid.

Initially, a $3 \mathrm{D}$ image in the NP was acquired. The volunteer was positioned in front of the $3 \mathrm{D}$ camera according to the user guide and asked to focus on the front mirror, while keeping a neutral face without any expression, during the first capture (Figure 2A). Subsequently, a disinfected eyelid hook was placed on the lower eyelid of the participant to pull down the lower eyelid and a second image in the DP was acquired under the same principle as the initial capture (Figure 2B). Afterwards, patients were asked to grade the discomfort during examination on a 4-point scale in none, mild, moderate, and severe discomfort. None indicated no symptoms, mild documented symptoms that were easily tolerated, moderate described the awareness of symptoms which were bothersome but tolerable, and severe matched symptoms that were only hard or not tolerable.

\section{Landmarks processing and data measurement}

In both positions (NP and DP), five basic landmarks were evaluated in each picture (Figure $3 A$ ), including the midpupil (Pc), medial (Ln) and lateral corneoscleral limbus $(\mathrm{Ll})$, endocanthion (En), and exocanthion (Ex) $(13,14)$. Six landmarks (Ln', Ln", Ll', Ll”, Ps, and Pi) were localized on the upper and lower eyelid margin based on the corresponding axis across the former three landmarks ( $\mathrm{Ln}, \mathrm{Ll}$, and $\mathrm{Pc}$ ). The Li landmark was localized on $\mathrm{Ll}$ corresponding to the axis across the $\mathrm{Pc}$; Pu represented the position of the lower punctum (Figure 3B).

Additionally, ten linear distances and two angles between the landmarks were calculated using the VAM software. All measurements for each image were repeated by the same observer after a $24-\mathrm{h}$ interval. The changes in the parameters between DP and NP were calculated using the mean values of both measurements (Table 1). 

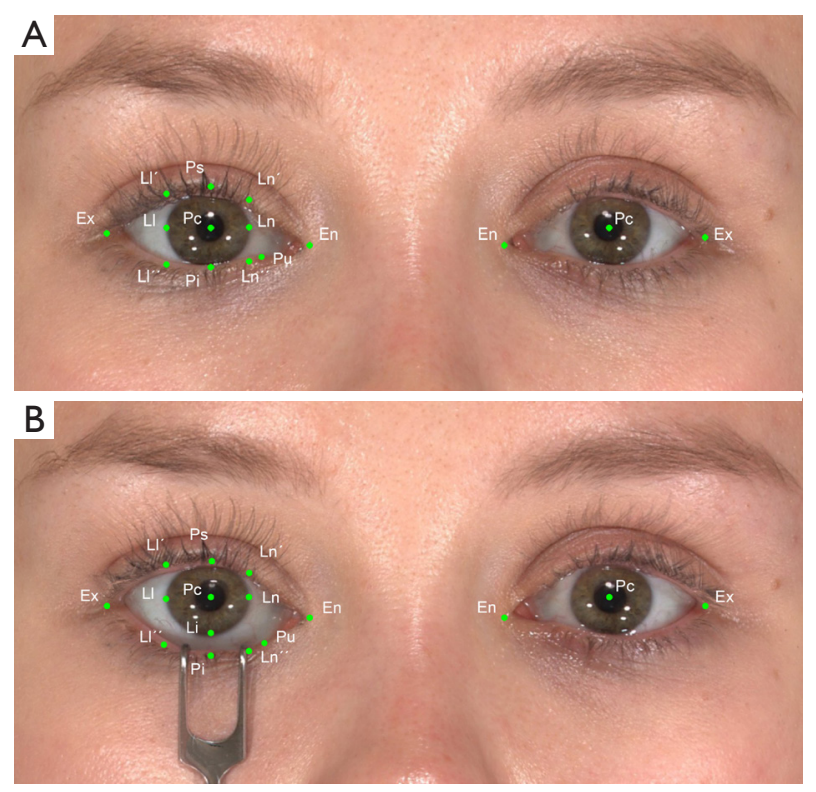

Figure 2 The 3D images of NP and DP (female, 23 years old). Five primary landmarks are localized in each image, including Pc, Ln and Ll, En and Ex. The landmarks of Ln' and Ln", $\mathrm{Ll}^{\prime}$ and $\mathrm{Ll}^{\prime \prime}, \mathrm{Ps}$ and $\mathrm{Pi}$ are localized on the lid margin corresponding to the axis across the Ln, $\mathrm{Ll}$ and $\mathrm{Pc}$, respectively; the landmark of Li was localized corresponding to the axis across the Pc on the corneoscleral limbus; Pu represented the position of lower punctum. (A) The 3D image of NP; (B) the 3D image of DP, with the eyelid hook, only the lower sclera of this participant exposed under the pull of the hook. 3D, three-dimensional; NP, neutral position; DP, distracted position; Pc, mid-pupil; Ln, the medial corneoscleral limbus; Ll, lateral corneoscleral limbus; En, endocanthion; Ex, exocanthion.
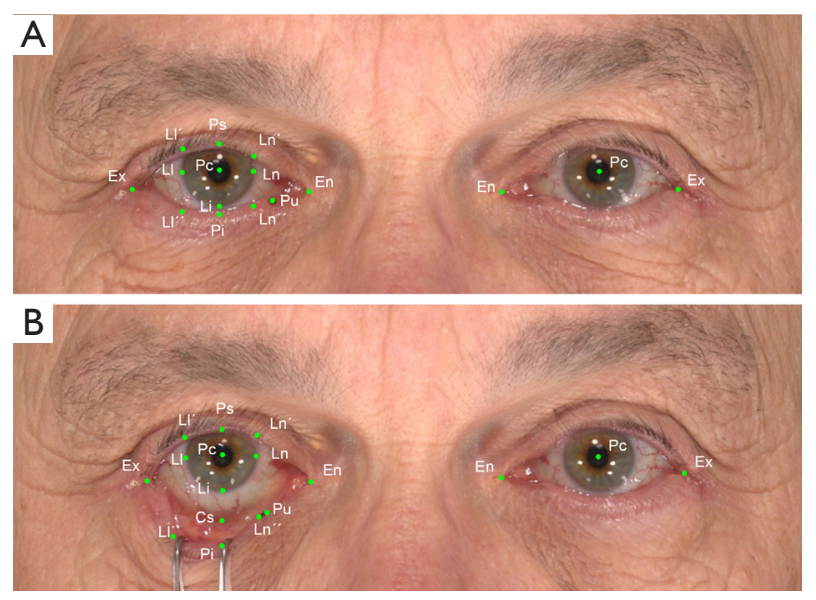

Figure 3 The 3D images of NP and DP (male, 65 years old). Five primary landmarks are localized in each image, including Pc, Ln and Ll, En and Ex. The landmarks of $\mathrm{Ln}^{\prime}$ and $\mathrm{Ln}^{\prime \prime}, \mathrm{Ll}^{\prime}$ and $\mathrm{Ll}^{\prime \prime}, \mathrm{Ps}$ and $\mathrm{Pi}$ are localized on the lid margin corresponding to the axis across the $\mathrm{Ln}$, $\mathrm{Ll}$ and $\mathrm{Pc}$, respectively; the landmark of Li was localized corresponding to the axis across the Pc on the corneoscleral limbus; Cs represents the point at the superior margin of the lower fornix conjunctiva vertically to Pc; Pu represented the position of lower punctum. (A) The 3D image of NP; (B) the 3D image of DP, with the eyelid hook, the lower fornix of this participant exposed under the pull of the hook. 3D, three-dimensional; NP, neutral position; DP, distracted position; Pc, mid-pupil; Ln, the medial corneoscleral limbus; Ll, lateral corneoscleral limbus; En, endocanthion; Ex, exocanthion. 
Table 1 Definition of landmarks, inter-landmark linear distances and angles

\begin{tabular}{|c|c|c|}
\hline Categories & Abbreviation & Definition \\
\hline \multirow{10}{*}{ Landmarks } & Ex & Exocanthion, outer commissure of the upper and lower eyelid margin \\
\hline & $\mathrm{Pc}$ & Pupillary center \\
\hline & Ps & Superior point vertical to $\mathrm{Pc}$ at the upper eyelid margin \\
\hline & $\operatorname{Ln}$ & Mid-nasal point of corneoscleral limbus \\
\hline & $\mathrm{Ln}^{\prime}$ & Superior point vertical to Ln at the upper eyelid margin \\
\hline & Ln" & Inferior point vertical to Ln at the lower eyelid margin \\
\hline & LI & Mid-temporal point of corneoscleral limbus \\
\hline & $\mathrm{Li}$ & Mid-inferior point of corneoscleral limbus \\
\hline & Cs & Point vertical to $\mathrm{Pc}$ at the superior margin of the lower fornix conjunctiva \\
\hline & $\mathrm{Pu}$ & Lower punctum point \\
\hline \multirow{6}{*}{$\begin{array}{l}\text { Inter-landmark linear } \\
\text { distances }\end{array}$} & IEn & Inter medial canthal distance, horizontal distance between En (left) and En (right) \\
\hline & $\mathrm{PcU}$ & Vertical height between $\mathrm{Pu}$ and $\mathrm{Pc}$ \\
\hline & IEx & Inter lateral canthal distance, horizontal distance between Ex (left) and Ex (right) \\
\hline & PcX & Vertical height between Ex and Pc \\
\hline & HPF & Horizontal palpebral fissure, horizontal distance between En and Ex \\
\hline & ConjE & Conjunctiva exposure, vertical distance between Cs and $\mathrm{Pi}$ \\
\hline \multirow[t]{2}{*}{ Inter-landmark angles } & LCA & Lateral canthal angle, angle between $\mathrm{LI}^{\prime}-\mathrm{Ex}-\mathrm{LI}^{\prime \prime}$ \\
\hline & MCA & Medial canthal angle, angle between Ln'-En-Ln" \\
\hline
\end{tabular}

\section{Statistical analyses}

$3 \mathrm{D}$ images for each participant were processed and measured twice with our landmark system by the same operator. All statistical analyses were performed using the SPSS version 23 software (IBM Corp., Armonk, NY, USA). Intraclass correlation coefficients (ICC) were used to evaluate the reliability of all the repeated measurements. The results were considered high reliability if the result was close to 1 , and low reliability, if the result was close to
0 (15). To assess the mean differences between the two sets of measurements, paired sample t-tests were performed for normally distributed data and paired Wilcoxon tests were used for non-normally distributed data. Multivariate general linear regression models were used to evaluate the differences between the sexes and the different age groups. $\mathrm{P}$ values $<0.05$ were considered statistically significant. GraphPad Prism 6 (GraphPad Software Inc., San Diego, CA, USA) was used to plot the corresponding bar graphs 
Table 2 Demographic characteristics of the participants

\begin{tabular}{|c|c|}
\hline Categories & Count \\
\hline \multicolumn{2}{|l|}{ Age (years old) } \\
\hline Range & $21-85$ \\
\hline Mean & $59.53 \pm 15.5$ \\
\hline \multicolumn{2}{|l|}{ Sex, n (\%) } \\
\hline Male & $50(53.2)$ \\
\hline Female & $44(46.8)$ \\
\hline Total & 94 \\
\hline \multicolumn{2}{|l|}{ Race/ethnicity, n (\%) } \\
\hline White/non-Hispanic & $92(97.9)$ \\
\hline Arabic & $2(2.1)$ \\
\hline \multicolumn{2}{|l|}{ Eyelid problems (cases) } \\
\hline Eyelid trauma & 0 \\
\hline Surgical procedures on the eyelid & 0 \\
\hline \multicolumn{2}{|l|}{ Discomfort level with eyelid hook } \\
\hline No discomfort & 80 \\
\hline Minor discomfort such as foreign body sensation & 9 \\
\hline $\begin{array}{l}\text { Moderate discomfort including redness, itching } \\
\text { or tearing }\end{array}$ & 5 \\
\hline $\begin{array}{l}\text { Severe discomfort including pain and severe } \\
\text { irritation }\end{array}$ & 0 \\
\hline
\end{tabular}

for the general linear models.

\section{Results}

The demographic features are presented in Table 2. A total of 94 participants [50 (53.2\%) men and 44 (46.8\%) women], aged 21-85 $(58.48 \pm 15.97)$ years, were included in this study. Ethnically, 92 participants were Caucasians and 2 were Arabians. Eighty-five percent of the participants $(n=80)$ had no discomfort, $10 \%(\mathrm{n}=9)$ reported minor discomfort, and only $5 \%(\mathrm{n}=5)$ of the participants indicated discomfort on a moderate level. None of the participant complaint severe discomfort. Table 3 shows the mean value of the two sets of measurements estimated using each $3 \mathrm{D}$ images for the ten inter-landmark linear distances [intermedial canthal distance (IEn), vertical height between punctum and pupillary center $(\mathrm{PcU})$, inter-lateral canthal distance (IEx), vertical height between exocanthion and pupillary center $(\mathrm{PcX})$, horizontal palpebral fissure (HPF), margin to reflex distance (MRD), inter-pupillary center distance (IPc), sclera exposure (ScE), vertical palpebral fissure (VPF), and conjunctiva exposure (ConjE)] and the two angles [lateral canthal angle (LCA) and medial canthal angle (MCA)].

Table 4 presents the ICC and mean differences between the two sets of measurements across all the inter-landmark linear distances and angles on each image. In NP, IEx and MRD had the highest ICC score of 0.97, whereas ConjE had the lowest score of 0.10 . The ICC results of the other nine measurements (PcU, HPF, IPc, ScE, VPF, IEn, PcX, LCA, and MCA) ranged between 0.55 and 0.88 . In DP, IEn, IPc, and VPF had the highest ICC score of 0.99, whereas HPF had the lowest ICC of 0.52 . The results of MRD and IEx ranked second with a score of 0.98 and the ICC of the other six measurements (PcU, PcX, LCA, MCA, $\mathrm{ScE}$, and ConjE) ranged between 0.79 and 0.90 . In NP, the mean values of IEn, PcU, PcX, LCA, MRD, IPc, VPF, and ConjE between the two repeated measurements of the same image had no significant differences $(\mathrm{P} \geq 0.05$, respectively). In contrast, those of the mean values of IEx, HPF, MCA, and $\mathrm{ScE}$ had statistically significant differences $(\mathrm{P}<0.05$, respectively). In DP, the mean values of IEx, LCA, MCA, and $\mathrm{ScE}$ between the two repeated measurements of the same image had statistically significant differences $(\mathrm{P}<0.05$, respectively), whereas the mean values of IEn, $\mathrm{PcU}$, MRD, IPc, VPF, PcX, HPF, and ConjE had no statistically significant differences $(\mathrm{P}>0.05$, respectively).

The mean differences of all the measurements between NP and DP images are shown in Table 5. The values of mean distracted displacements were as follows: ConjE*, $3.24 \pm 2.61 \mathrm{~mm}$; IEn ${ }^{*}, 0.12 \pm 0.98 \mathrm{~mm}, \mathrm{PcU}^{*}, 3.95 \pm 1.51 \mathrm{~mm}$; $\mathrm{PcX}^{*}, 1.05 \pm 1.14 \mathrm{~mm}$; MRD* $5.50 \pm 1.53 \mathrm{~mm}$; $\mathrm{IPc}^{*}$, $0.02 \pm 0.27 \mathrm{~mm}$; ScE ${ }^{*}, 4.61 \pm 1.38 \mathrm{~mm}$; and $\mathrm{VPF}^{*}, 4.92 \pm 1.77 \mathrm{~mm}$. The IEx* decreased by $1.02 \pm 1.15 \mathrm{~mm}^{*}$ and the $\mathrm{HPF}^{*}$ decreased by $1.58 \pm 3.11 \mathrm{~mm}$. The $\mathrm{LCA}^{*}$ and the MCA* increased between BP and DP by $26.67 \pm 13.83$ degree and $18.31 \pm 9.87$ degree, respectively.

Multivariate general linear regression models were used to analyse the displacement between NP and DP across the sexes and nine age groups ( 21 to 30,31 to 40,41 to 50 , 51 to 55,56 to 60,61 to 65,66 to 70,71 to 75 , and 76 to 85 years); the results are shown in Table 6 and Figure 4. Except the $\mathrm{PcU}^{*}$, which showed a significant difference $(\mathrm{P}=0.02)$ in different age groups, all other measurements had no significant difference among the nine age groups $(\mathrm{P}>0.05$, respectively). For the $\mathrm{PcU}^{*}$, the 21-30-year group and the 31-40-year group had a smaller magnitude of change than 51-55-year, 56-60-year, 61-65-year and 71-75-year groups 
Table 3 Means and standard deviations (SD) across all measurements in neutral and distracted position

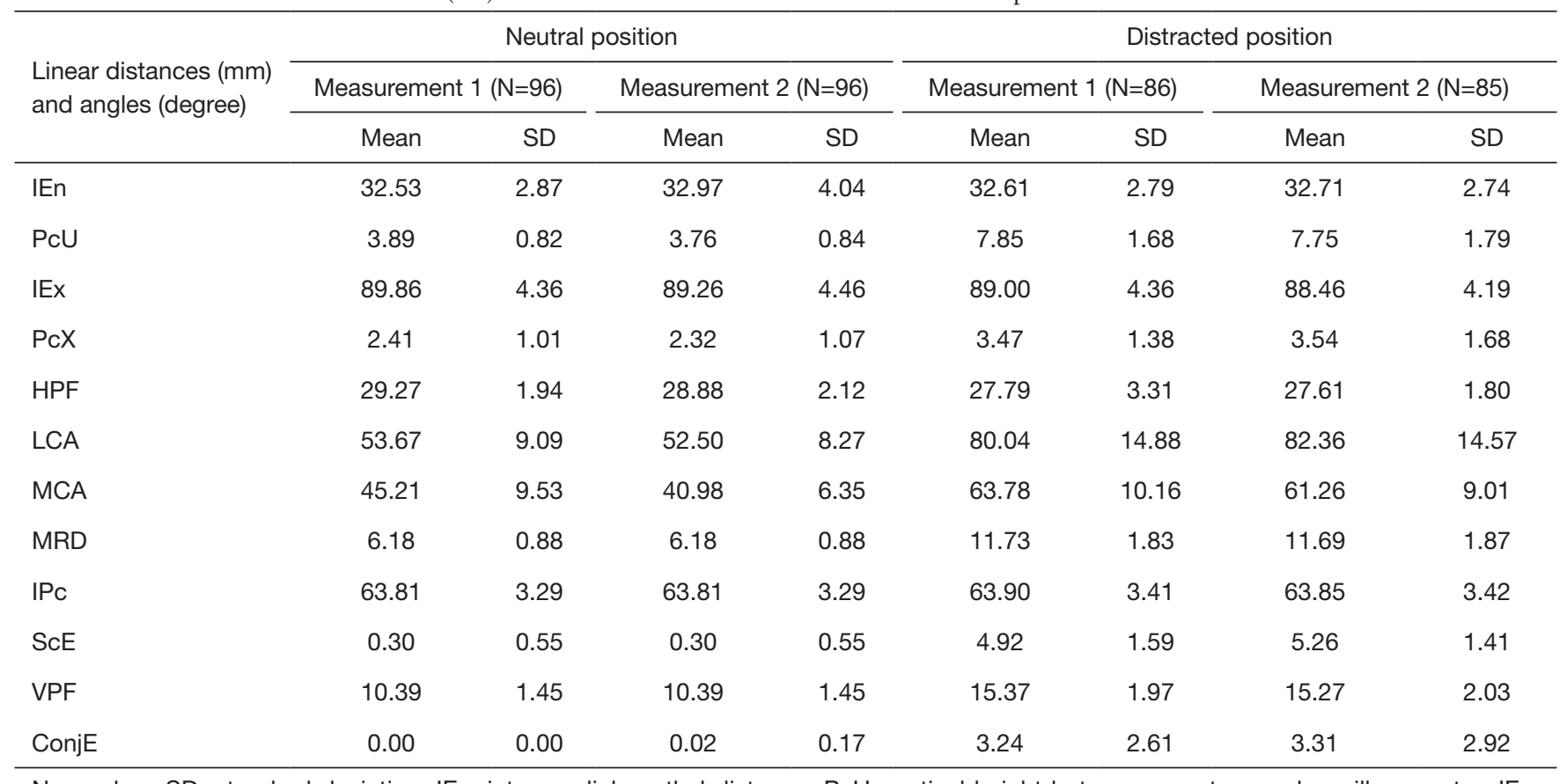

N, number; SD, standard deviation; IEn, inter-medial canthal distance; PcU, vertical height between punctum and pupillary center; IEx, inter-lateral canthal distance; PcX, vertical height between exocanthion and pupillary center; HPF, horizontal palpebral fissure; MRD, margin to reflex distance; IPc, inter-pupillary center distance; ScE, sclera exposure; VPF, vertical palpebral fissure; ConjE, conjunctiva exposure; LCA, lateral canthal angle; MCA, medial canthal angle.

$(\mathrm{P}<0.05$, respectively). The distracted displacement values of $\mathrm{PcU}^{*}, \mathrm{LCA}^{*}, \mathrm{MRD}^{*}, \mathrm{ScE}^{*}$, and ConjE* had a statistically significant difference $(\mathrm{P}<0.05$, respectively) between both the sexes, with the displacement among men demonstrating a greater magnitude of change than that among women. The distracted displacements of IEn* ${ }^{*} \mathrm{IEx}^{*}, \mathrm{PcX}^{*}, \mathrm{HPF}^{*}$, $\mathrm{MCA}^{*}, \mathrm{IPc}^{*}$, and $\mathrm{VPF}^{*}$ showed no significant difference between men and women $(\mathrm{P}>0.05$, respectively).

\section{Discussion}

In this study, we aimed to investigate the possibility of indirectly evaluating LET using a 3D imaging system, VECTRA M3 stereophotogrammetry device. Our study findings confirmed the feasibility of using a digital imaging system to accurately perform DT (12). In addition, this study was the first to use a series of detailed periocular landmarks in the DT; this study is also the first to validate the feasibility of the corresponding inter-landmark measurements. Furthermore, we utilized a standard weighted eyelid hook to substitute the conventional, manual grasping of the lower eyelid by the examiner's thumb and index finger. To our best knowledge, this study is also the first to assess LET.

Recently, Stuchi et al. (12) were the first to validate the reliability of a $3 \mathrm{D}$ imaging system to quantitatively evaluate lower eyelid laxity. However, they only investigated one parameter, the change in vertical distance of the lower eyelid between NP and DP, which is insufficient to fully assess the applicability of this method. Based on the successful application of the landmarks system on the periocular region $(16,17)$, fourteen landmarks, ten interlandmark linear distances, and two inter-landmark angles were investigated in this study.

In the previous studies $(10,11,16,17)$, the $3 \mathrm{D}$ imaging system was mainly used on neutral expression surfaces with the landmarks system. To the best of our knowledge, this is the first investigation to apply $3 \mathrm{D}$ stereophotogrammetry on both neutral surfaces and surfaces with the stainless eyelid hook. In our study, except for ConjE (0.10) in NP and HPF (0.52) in DP, the ICC score for all the other 22 measurements (among the 24 measurements) was more than 0.76 , which indicated that our study had reliability ranging from "good" to "very good" for repeated measurements 
Table 4 Mean difference and paired t-test between two sets of measurements for all the linear distances and angles

\begin{tabular}{|c|c|c|c|c|c|c|}
\hline Categories & Linear distances and angles & ICC (95\% CI) & Mean & SD & $\mathrm{t}$ & $\mathrm{P}$ \\
\hline \multirow{8}{*}{$\begin{array}{l}\text { Neutral position } \\
(\mathrm{N}=94)\end{array}$} & IEn & $0.76(0.63-0.84)$ & -0.43 & 3.11 & -1.35 & 0.18 \\
\hline & $\mathrm{PcU}$ & $0.85(0.73-0.97)$ & 0.14 & 0.70 & 2.01 & 0.05 \\
\hline & IEx & 0.97 (0.93-0.99) & 0.71 & 1.29 & 5.38 & 0.00 \\
\hline & PcX & $0.78(0.69-0.87)$ & 0.10 & 0.62 & 1.62 & 0.11 \\
\hline & LCA & $0.79(0.68-0.86)$ & 1.35 & 7.06 & 1.86 & 0.07 \\
\hline & MCA & $0.55(0.28-0.71)$ & 4.20 & 8.67 & 4.72 & 0.00 \\
\hline & MRD & $0.97(0.95-0.98)$ & -0.03 & 0.32 & -1.02 & 0.31 \\
\hline & IPc & $0.84(0.75-0.89)$ & 0.32 & 3.01 & 1.03 & 0.31 \\
\hline \multirow{7}{*}{$\begin{array}{l}\text { Distracted position } \\
(\mathrm{N}=83)\end{array}$} & IEn & 0.99 (0.98-0.99) & -0.03 & 0.63 & -0.48 & 0.63 \\
\hline & $\mathrm{PcU}$ & $0.85(0.80-0.90)$ & 0.14 & 1.15 & 1.13 & 0.26 \\
\hline & IEx & $0.98(0.96-0.99)$ & 0.54 & 0.99 & 4.97 & 0.00 \\
\hline & PcX & $0.79(0.69-0.86)$ & -0.05 & 1.26 & -0.33 & 0.74 \\
\hline & HPF & $0.52(0.26-0.69)$ & 0.14 & 3.04 & 0.44 & 0.66 \\
\hline & LCA & $0.87(0.79-0.91)$ & -2.53 & 10.00 & -2.32 & 0.02 \\
\hline & MCA & $0.80(0.67-0.87)$ & 2.65 & 7.68 & 3.17 & 0.00 \\
\hline
\end{tabular}

ICC, intraclass correlation coefficients; Cl, confidence interval; N, number; SD, standard deviation; IEn, inter-medial canthal distance; PcU, vertical height between punctum and pupillary center; IEx, inter-lateral canthal distance; PcX, vertical height between exocanthion and pupillary center; HPF, horizontal palpebral fissure; MRD, margin to reflex distance; IPc, inter-pupillary center distance; ScE, sclera exposure; VPF, vertical palpebral fissure; ConjE, conjunctiva exposure; LCA, lateral canthal angle; MCA, medial canthal angle.

on the same image for both NP and DP. Furthermore, the score of MRD $(18,19)$ showed high reliability for both NP (0.97) and DP (0.98) and was consistent with the previous research (12), which also proved that our novel method is a potential standard tool to measure the LET based on $3 \mathrm{D}$ technology.

Although the measurement of ConjE (0.90) obtained in the DP had very good reliability, its score $(0.10)$ was extremely low in the neutral expression images, which might be because, in normal eyes, there is generally no or very little conjunctival exposure as compared to the mean ConjE of the two measurements in our study $(0.00 \pm 0.00$ and $0.02 \pm 0.17 \mathrm{~mm}$, respectively). Hence, the application of ConjE was not recommended in NP due to the reliability of Vectra and the landmarks system could be limited to a mean value of less than $0.2 \mathrm{~mm}$. However, the good ICC score of ConjE in DP might indicate the reliability of the landmark on the sclera. As the landmark has never been investigated on the sclera in previous studies, our research might serve as a stepping-stone for further research. Additionally, the 
Table 5 Mean differences between neutral and distracted position in different age groups and genders

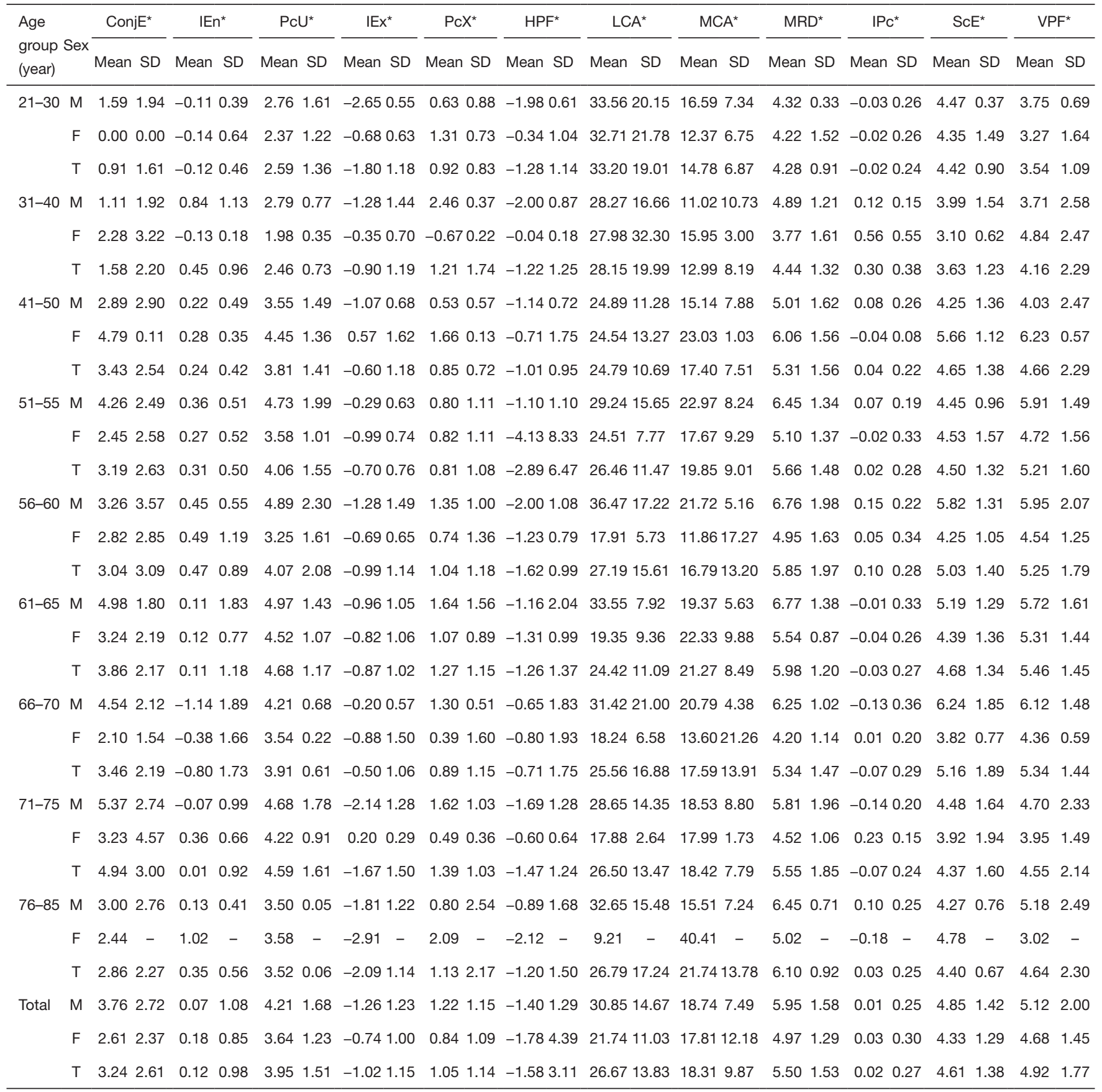

*, increment of each variable between neutral and distracted position. SD, standard deviation; IEn, inter-medial canthal distance; PcU, vertical height between punctum and pupillary center; IEx, inter-lateral canthal distance; PcX, vertical height between exocanthion and pupillary center; HPF, horizontal palpebral fissure; MRD, margin to reflex distance; IPc, inter-pupillary center distance; ScE, sclera exposure; VPF, vertical palpebral fissure; ConjE, conjunctiva exposure; LCA, lateral canthal angle; MCA, medial canthal angle. 
Table 6 Significant test for the difference between neutral and distracted position across genders and all the age groups

\begin{tabular}{|c|c|c|c|c|c|c|}
\hline \multirow{2}{*}{$\begin{array}{l}\text { Linear distances } \\
\text { and angles }\end{array}$} & \multicolumn{2}{|c|}{$\begin{array}{l}\text { Levene's test for equality of } \\
\text { error variances }^{a}\end{array}$} & \multicolumn{4}{|c|}{ Tests of between-subjects effects } \\
\hline & $\mathrm{F}$ & $\mathrm{P}$ & $\mathrm{F}$ & $P$ & $\mathrm{~F}$ & $\mathrm{P}$ \\
\hline $\mathrm{IEn}^{*}$ & 2.32 & 0.01 & 1.48 & 0.18 & 0.16 & 0.69 \\
\hline $\mathrm{PcU}^{*}$ & 1.22 & 0.27 & 2.57 & 0.02 & 5.04 & 0.03 \\
\hline $\mathrm{PcX}^{*}$ & 0.87 & 0.61 & 0.31 & 0.96 & 2.10 & 0.15 \\
\hline $\mathrm{HPF}^{*}$ & 0.55 & 0.92 & 0.48 & 0.87 & 0.10 & 0.76 \\
\hline LCA $^{*}$ & 1.55 & 0.10 & 0.33 & 0.95 & 9.88 & $<0.01$ \\
\hline $\mathrm{MCA}^{*}$ & 0.91 & 0.57 & 0.62 & 0.76 & 0.46 & 0.50 \\
\hline $\mathrm{ScE}^{*}$ & 0.63 & 0.85 & 0.86 & 0.56 & 4.09 & 0.05 \\
\hline $\mathrm{VPF}^{*}$ & 0.67 & 0.82 & 1.31 & 0.25 & 3.14 & 0.08 \\
\hline ConjE $^{\star}$ & 1.14 & 0.34 & 1.76 & 0.10 & 4.19 & 0.04 \\
\hline
\end{tabular}

${ }^{a}$, tests the null hypothesis that the error variance of the dependent variable is equal across groups; *, increment of each variable between neutral and distracted position. IEn, inter-medial canthal distance; PcU, vertical height between punctum and pupillary center; IEx, inter-lateral canthal distance; PcX, vertical height between exocanthion and pupillary center; HPF, horizontal palpebral fissure; MRD, margin to reflex distance; IPc, inter-pupillary center distance; ScE, sclera exposure; VPF, vertical palpebral fissure; ConjE, conjunctiva exposure; LCA, lateral canthal angle; MCA, medial canthal angle.

ICC scores of IPc were very good in both NP (0.84) and DP (0.99), which revealed that the landmarks on the cornea centre had good reliability and were highly consistent with the previous studies $(14,16,17)$. Hence, although problems concerning reflection could affect the picture quality, the system of automatic and manual registration of landmarks with Vectra was proven capable of determining the position of the cornea and sclera precisely.

The results of the mean difference between the two sets of measurements on the same NP image indicated that the MRD, IEn, PcX, IPc, VPF, and ConjE distances, as well as the angle of LCA conferred better reliability in repeated measurements by the same examiner. This observation was also consistent with those reported by previous studies $(12,14,16,17,20,21)$. Although the result of PcU, IEx, HPF and ScE distances, as well as the angle of MCA in NP had a low intra-rater reliability, the differences in the measurements was within $1 \mathrm{~mm}$ (1 unit), which had no clinical significance and were consistent with the results of previous studies $(14,16,17,20,21)$. In the DP, the majority of the inter-landmark distances had no significant difference between two measurement. Only the IEx and ScE distances had statistically significant differences $(0.54$ and $-0.31 \mathrm{~mm}$, respectively); however, this result had no clinical significance. However, for the inter-landmark angle validation, significant difference was found between the two measurements. This difference might be due to the influence of position changes caused by the examiner's inability to accurately localize the landmarks on the lower eyelid margin and also due to the interference of the upper and lower eyelashes.

Although several studies have attempted to evaluate the eyelid tension with different methods, including the use of piezoresistive sensor (22), lid speculum (6), and eyelid tensiometer (4-6), none of these methods have been used in clinical settings due to subjects' discomfort and lack of coordination. Conventionally, the horizontal lower eyelid laxity was evaluated by grasping the lower eyelid outward using the thumb and index finger and measuring the distracted distance from the globe (12). However, with this method, it is difficult to ensure uniformity in the pulling strength even with the same operator. According to the law of energy conservation in physics, the eyelid tension 


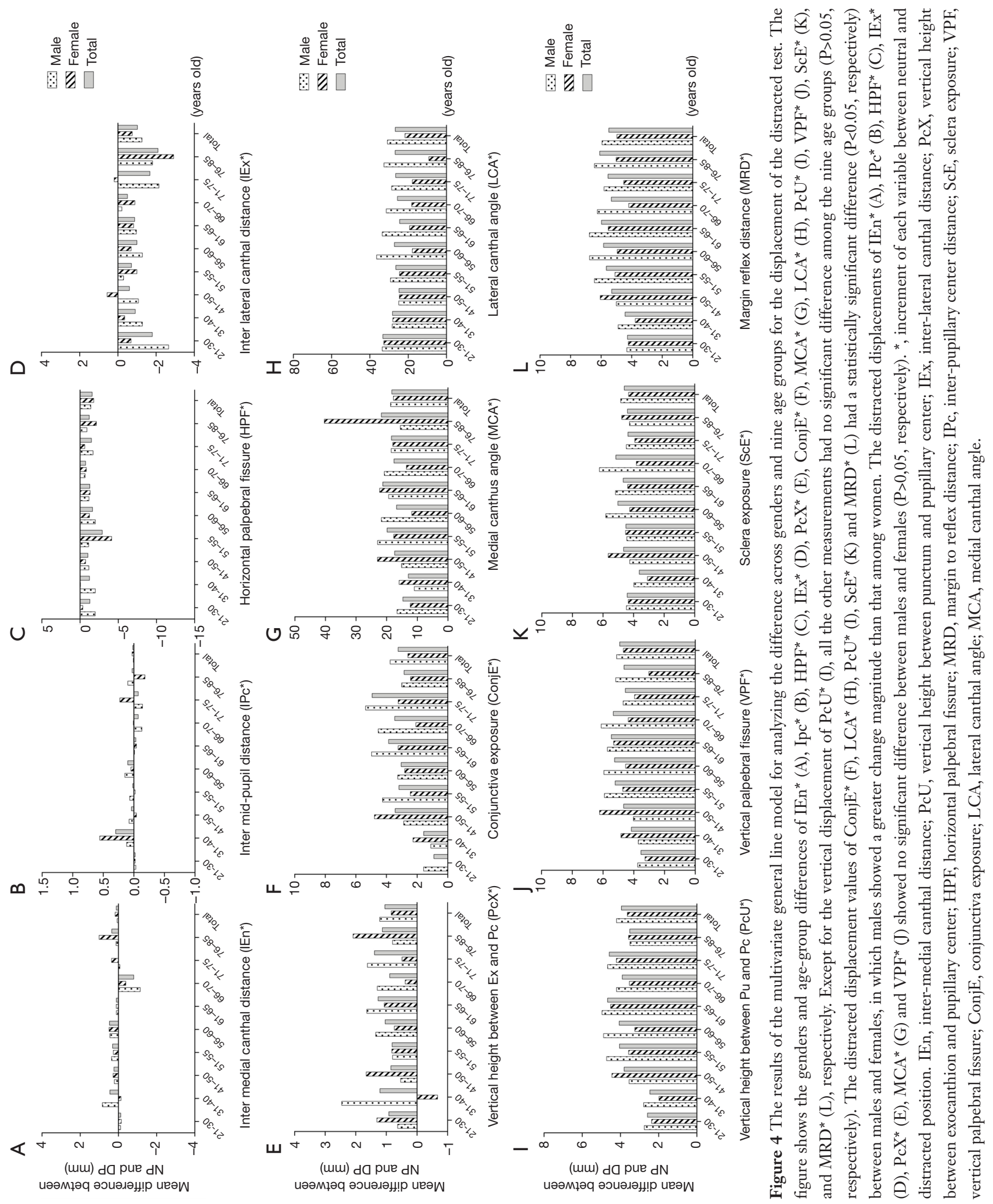


can be reasonably evaluated by hanging a weighted eyelid hook on the lower eyelid. Due to individual discrepancies in the supporting tissue of the lower eyelid, the same eyelid hook with the same quality could cause different degrees of maximum displacements of the lower eyelid. Hence, this displacement can be represented as LET. In our study, most participants did not feel any discomfort or minor discomfort, except very few sensitive individuals who experienced redness, tearing or difficult cooperating.

Most surgeons distract the mid-margin of the eyelid outward from the globe and measure the distance between the eyeball and the farthest margin of the lower lid, i.e., conventional DT (12). Whereas in the 3D-DT, we pulled the lower eyelid downwards with the weight. Although the lower eyelid behaves differently in the outward pull (DT) and downward pull (3D-DT) test, both results might be used equally to evaluate an existing laxity before and after lower lid blepharoplasty, as the lower eyelid is pulled away from the eyeball and the laxity could be assessed by calculating the eyelid displacement from the original position in millimeter. However, in traditional DT, it is quite difficult to ensure an accurate original position of the eyelid margin on the eyeball surface (12). In our current study using a 3D camera, the eyelid displacement was calculated based on the MRD $(18,19)$ and the eyelid displacement could be easily recognized by pulling the lid margin downward on the front view. Hence, our method could be considered as a modified DT and have the same evaluating value as the traditional DT.

The results of the multivariate general linear regression models showed that nearly all the displacements between the NP and DP had no significant differences between the young and old age groups. This result was highly consistent with those reported previously, which investigated the feasibility of DT in normal subjects with different age groups (19). Histopathologic analysis of the eyelids with senile ectropion or entropion revealed increased adipose tissue in the distal tarsus, laxity of the canthal tendons, degeneration of the skin and collagen, and elastosis of the tarsal plate (23). These anatomical alterations might also support the hypothesis that the aging process may yield a higher DT value in the older population, especially in patients with involutional ectropion (24). However, age is not an isolated factor for the development of eyelid laxity or even ectropion, as the metabolic and anatomical alterations may also be the influence factors (25), and not all the older individuals develop pathological changes (26).

Moreover, the average displacement of the MRD (which represents LET) was $5.50 \pm 1.53 \mathrm{~mm}$ and showed also a high consistence with the previously reported normal reference value $(6.96 \mathrm{~mm})$ obtained using the conventional DT $(18,19)$. Furthermore, the MRD displacement showed a significant difference between men and women, with a relatively high magnitude of displacement observed among men $(5.95 \pm 1.58 \mathrm{~mm})$ than in women $(4.97 \pm 1.29 \mathrm{~mm})$. This result was also consistent with those reported previously (19).

To the best of our knowledge, this is the first study to investigate eyelid displacement using a standardized DT with 3D stereophotogrammetry and utilizing detailed periocular landmarks on acquired 3D images. No corresponding reference value was found for the displacement of IEn, $\mathrm{PcU}$, IEx, PcX, HPF, LCA, MCA, IPc, ScE, VPF, and ConjE. We believe that the results reported herein could be considered as the reference values in future studies.

A potential limitation of the study is that individuals with pathological changes for the lower eyelid were not included. Thus, this patient group should be included as a comparative group in future studies.

In summary, our study demonstrated a high agreement with the previously reported reference value and confirmed the feasibility of using a novel, standardized DT and landmarks system to evaluate LET based on the 3D stereophotogrammetry. Moreover, the results build the basis for future research. This novel and simple method might be utilized in routine clinical settings preoperatively, specifically, before plastic and reconstructive surgeries of the eyelids.

\section{Acknowledgments}

The authors thank Editage (www.editage.com) for English language editing.

Funding: This work was supported by the Fundamental Research Funds for the Central Universities (grant No. 2021FZZX005-15), the Koeln Fortune Program/Faculty of Medicine, University of Cologne, Germany (grant No. 2680148101) and the State Scholarship Fund from China Scholarship Council (grant No. 201708080141) to Yongwei Guo; National Natural Science Foundation of China (grant No. 81970834) and Science and Technology Commission of Shanghai Municipality (grant No. 19441900800) to Ming Lin; GEROK program of the University of Cologne to Alexander C. Rokohl.

\section{Footnote}

Conflicts of Interest: All authors have completed the ICMJE 
uniform disclosure form (available at http://dx.doi. org/10.21037/qims-20-1016). The authors have no conflicts of interest to declare.

Ethical Statement: The authors are accountable for all aspects of the work in ensuring that questions related to the accuracy or integrity of any part of the work are appropriately investigated and resolved. The study was conducted in accordance with the Declaration of Helsinki (as revised in 2013). The study was approved by the institutional ethics board of the University of Cologne and informed consent was taken from all individual participants.

Open Access Statement: This is an Open Access article distributed in accordance with the Creative Commons Attribution-NonCommercial-NoDerivs 4.0 International License (CC BY-NC-ND 4.0), which permits the noncommercial replication and distribution of the article with the strict proviso that no changes or edits are made and the original work is properly cited (including links to both the formal publication through the relevant DOI and the license). See: https://creativecommons.org/licenses/by-nc-nd/4.0/.

\section{References}

1. Branham G, Holds JB. Brow/Upper lid anatomy, aging and aesthetic analysis. Facial Plast Surg Clin North Am 2015;23:117-27.

2. Triana RJ Jr, Larrabee WF Jr. Lower eyelid blepharoplasty: the aging eyelid. Facial Plast Surg 1999;15:203-12.

3. Pessa JE, Desvigne LD, Lambros VS, Nimerick J, Sugunan B, Zadoo VP. Changes in ocular globe-toorbital rim position with age: implications for aesthetic blepharoplasty of the lower eyelids. Aesthetic Plast Surg 1999;23:337-42.

4. Fu ZX, Zhang XR, Zhang ZY, Li QS, Wang HM, Xiang MH, Han ZM. Lower eyelid tension in young adults determined by a simple lid tensiometer. Cornea 2014;33:518-20.

5. Vihlen FS, Wilson G. The relation between eyelid tension, corneal toricity, and age. Invest Ophthalmol Vis Sci 1983;24:1367-73.

6. Wilson G, Bell C, Chotai S. The effect of lifting the lids on corneal astigmatism. Am J Optom Physiol Opt 1982;59:670-4.

7. Ehrmann K, Francis I, Stapleton F. A novel instrument to quantify the tension of upper and lower eyelids. Cont Lens Anterior Eye 2001;24:65-72.
8. Kim J, Lee SM, Choi YJ, Lee MJ. Estimation of Eyelid Pressure Using a Blepharo-Tensiometer in Patients with Functional Nasolacrimal Duct Obstruction. J Ophthalmol 2018;2018:8792102.

9. Villano ME, Leake DS, Jacono AD, Quatela VC. Effects of endoscopic forehead/midface-lift on lower eyelid tension. Arch Facial Plast Surg 2005;7:227-30.

10. Dindaroğlu F, Kutlu P, Duran GS, Görgülü S, Aslan E. Accuracy and reliability of 3D stereophotogrammetry: A comparison to direct anthropometry and 2D photogrammetry. Angle Orthod 2016;86:487-94.

11. Wong JY, Oh AK, Ohta E, Hunt AT, Rogers GF, Mulliken JB, Deutsch CK. Validity and reliability of craniofacial anthropometric measurement of 3D digital photogrammetric images. Cleft Palate Craniofac J 2008;45:232-9.

12. Stuchi DPA, Rossato J, Bocaccio FJL, Procianoy F. Intraand interobserver reliability of a modified distraction test based on digital images to assess lower eyelid horizontal tension. Arq Bras Oftalmol 2020;83:127-31.

13. Guo Y, Liu J, Ruan Y, Rokohl AC, Hou X, Li S, Jia R, Koch KR, Heindl LM. A novel approach quantifying the periorbital morphology: A comparison of direct, 2-dimensional, and 3-dimensional technologies. J Plast Reconstr Aesthet Surg 2020. [Epub ahead of print]. doi: 10.1016/j.bjps.2020.12.003.

14. Guo Y, Schaub F, Mor JM, Jia R, Koch KR, Heindl LM. A Simple Standardized Three-Dimensional Anthropometry for the Periocular Region in a European Population. Plast Reconstr Surg 2020;145:514e-523e.

15. Metzler P, Sun Y, Zemann W, Bartella A, Lehner M, Obwegeser JA, Kruse-Gujer AL, Lubbers HT. Validity of the 3D VECTRA photogrammetric surface imaging system for cranio-maxillofacial anthropometric measurements. Oral Maxillofac Surg 2014;18:297-304.

16. Guo Y, Hou X, Rokohl AC, Jia R, Heindl LM. Reliability of Periocular Anthropometry: A Comparison of Direct, 2-Dimensional, and 3-Dimensional Techniques. Dermatol Surg 2020;46:e23-31.

17. Guo Y, Rokohl AC, Schaub F, Hou X, Liu J, Ruan Y, Jia R, Koch KR, Heindl LM. Reliability of periocular anthropometry using three-dimensional digital stereophotogrammetry. Graefes Arch Clin Exp Ophthalmol 2019;257:2517-31.

18. Putterman AM. Margin reflex distance (MRD) 1, 2, and 3. Ophthalmic Plast Reconstr Surg 2012;28:308-11.

19. Milbratz-Moré GH, Pauli MP, Lohn CLB, Pereira FJ, Grumann AJ. Lower Eyelid Distraction Test: New Insights 
on the Reference Value. Ophthalmic Plast Reconstr Surg 2019;35:574-7.

20. Heike CL, Cunningham ML, Hing AV, Stuhaug E, Starr JR. Picture perfect? Reliability of craniofacial anthropometry using three-dimensional digital stereophotogrammetry. Plast Reconstr Surg 2009;124:1261-72.

21. Aldridge K, Boyadjiev SA, Capone GT, DeLeon VB, Richtsmeier JT. Precision and error of threedimensional phenotypic measures acquired from $3 \mathrm{dMD}$ photogrammetric images. Am J Med Genet A 2005;138A:247-53.

22. Shaw AJ, Collins MJ, Davis BA, Carney LG. Eyelid pressure and contact with the ocular surface. Invest Ophthalmol Vis Sci 2010;51:1911-7.

Cite this article as: Hou $\mathrm{X}$, Rokohl AC, Meinke MM, Li S, Liu J, Fan W, Lin M, Jia R, Guo Y, Heindl LM. A novel standardized distraction test to evaluate lower eyelid tension using three-dimensional stereophotogrammetry. Quant Imaging Med Surg 2021;11(8):3735-3748. doi:10.21037/qims-20-1016
23. Kocaoglu FA, Katircioglu YA, Tok OY, Pulat H, Ornek F. The histopathology of involutional ectropion and entropion. Can J Ophthalmol 2009;44:677-9.

24. Damasceno RW, Avgitidou G, Belfort R Jr, Dantas PE, Holbach LM, Heindl LM. Eyelid aging: pathophysiology and clinical management. Arq Bras Oftalmol 2015;78:328-31.

25. Jyothi SB, Seddon J, Vize CJ. Entropion-ectropion: the influence of axial globe length on lower eyelid malposition. Ophthalmic Plast Reconstr Surg 2012;28:199-203.

26. Mitchell P, Hinchcliffe P, Wang JJ, Rochtchina E, Foran S. Prevalence and associations with ectropion in an older population: the Blue Mountains Eye Study. Clin Exp Ophthalmol 2001;29:108-10. 